\title{
A Deformable Elastic Matching Model for Handwritten Chinese Character Recognition
}

\author{
Lian-wen Jin \\ Department of Computer Science \\ The University of Hong Kong \\ Pokfulam Rd., Hong Kong
}

\author{
Kwok-ping Chan \\ Department of Computer Science \\ The University of Hong Kong \\ Pokfulam Rd., Hong Kong
}

Bing-zheng $\mathrm{Xu}$

The Institute of Radio Engineering and Automation

South China University of Technology

GuangZhou, P.R. China

\begin{abstract}
This paper describes a deformable elastic matching approach to Handwritten Chinese Character Recognition(HCCR). Handwritten character is regarded as a kind of deformable object, with elastic property. For the same category of character, we assume that different handwriting variations share the same topological structure, but may differ in shape details. The variations between different handwriting characters are modelled by a set of stroke displacement vectors(SDV). According to the SDV derived, a model character is deformed gradually, in an effort to transform itself much closer to an input character. Experiments show that the proposed elastic matching model can efficiently deal with local shape changes and variations between characters.
\end{abstract}

Key words: Elastic matching, deformation, handwritten Chinese character recognition, displacement vector, flexible template.

\section{Introduction}

Object recognition and image interpretation using rigid model is well established. Objects under such modelling only undergo translation and rotation. However, in many practical situations, real world objects not only translate, rotate, but also scale and may even be deformable. In such case, rigid model is less powerful. Recently, modelling of objects as deformable elastic bodies has gained more attention in computer vision and image processing[1]-[8]. Many deformable models for image analysis have been established, such as the Rubber Mask[1], Iterative Elastic Matching Technique[2], Energy Constraint Deformable Model[3], Point Distribution Model [5]-[6], and Active Contour Model[4].

Handwritten Character Recognition has been an attractive and challenging problem in the field of pattern recognition and computer vision for many years [9]. Handwritten Chinese character recognition is even more difficult due to the complex structure of Chinese characters, large vocabulary, many mutual similarity and great variability with different handwriting styles. From our point of view, handwritten Chinese character can be regarded as a kind of deformable.object. Although there are large variations in the same class of character for different styles of handwriting, the basic topological structures of them are the same. Different handwriting variations can be regarded as the distorted versions from a deformable model. Therefore, it is natural to model handwritten character with deformable template, which will be good enough to represent the essential topological structure of a class of characters.

Some earlier works of using deformable model in handwriting recognition were reported by J.M Bertille [10] and T. Wakahara [11]. In [10], Bertille applied an elastic matching technique to handwritten digit recognition. An unknown pattern is distorted under some constraints (Energy Function) to establish a matching with the corresponding prototype. A cost function is defined to measure the dissimilarity between the unknown character and the prototype. 
However, the validity of this approach depends on the proper design of the energy function and cost function. For handwritten Chinese character, it is not easy to find such functions. In [11], Wakahara proposed a robust deformable matching approach to handwritten numeral recognition. A Local Affine Transform (LAT) is employed to model the displacement of each points in a character image. A model character is distorted by the LAT operations so as to yield the best match to an input character. However, the structure of Chinese is so complex that it may not be sufficient to model distortions just by LAT. Moreover, the number of pixel points of Chinese character is much more than that of numerals, therefore there would be heavy computation involved when this method is applied to Chinese character recognition.

In this paper, we will propose a stroke-based deformable elastic matching technique for handwritten Chinese character recognition(HCCR). Handwritten Chinese character is regarded as a kind of deformable object, with elastic property. Each class of character is modelled with a flexible template. When matching an unknown character with a template, a set of Displacement Vectors(DVs) is first derived from local match of these two characters, then the template is gradually deformed according to the displacement vectors, in an effort to be much closer to the unknown character. As a Chinese character is written stroke by stroke, we will determine the displacement vector based on strokes of a character, rather than pixel points of a character, hence making the matching process much faster and more efficient than a pixel-based matching scheme, like that in [10] and [11]. After deformation process of all templates, the one which gives the best match will be selected as the recognition result.

In section 2, we will give details on how to determine the stroke-based DV. In section 3 we apply the proposed method to handwritten Chinese character recognition. Section 4 gives the conclusion remark.

\section{Stroke-based deformable elastic matching}

\subsection{Stroke representation of hand- written Chinese character}

A handwritten Chinese character is represented by a set of strokes:

$$
C=\left\{s_{1}, s_{2}, \ldots, s_{m}\right\}
$$

where $s_{i}$ is the ith stroke of character $C$. Stroke is a line segment in a character, thus it is natural to represent the stroke by it's two end-points:

$$
s_{i}=\left\{\overrightarrow{p^{s}}, \overrightarrow{p^{e}}\right\}=\left\{\left(x_{i}^{s}, y_{i}^{s}\right),\left(x_{i}^{e}, y_{i}^{e}\right)\right\}
$$

\subsection{Stroke Displacement Vector}

Given a model character $M$ and an unknown character T:

$$
M=\left\{s_{1}, s_{2}, \ldots, s_{m}\right\} \quad, \quad T=\left\{t_{1}, t_{2}, \ldots, t_{n}\right\}
$$

where $s_{i}=\left\{\overrightarrow{p_{1}}, \overrightarrow{p_{2}}\right\}$ is the ith stroke in $\mathrm{M}$ and $t_{j}=$ $\left\{\overrightarrow{q_{1}}, \overrightarrow{q_{2}}\right\}$ is the $j t h$ stroke in $\mathrm{T}$. Matching character $M$ with $T$ is equivalent to matching strokes of them accordingly. Variation and distortion between two characters can be described by a set of Displacement Vectors (DVs) derived from each stroke, as demonstrated in figure 1.

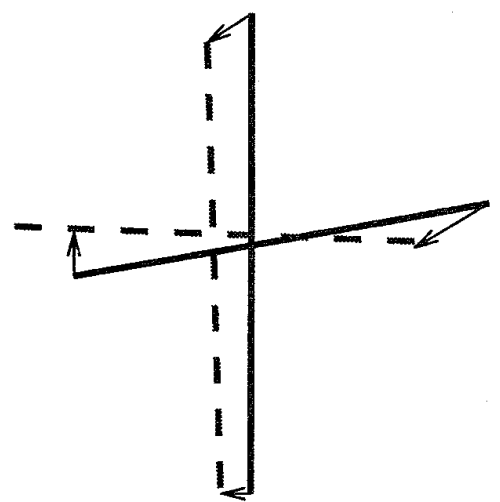

Figure 1: A simple example to illuminate Displacement Vectors between two Chinese characters "ten"

Displacement vector from stroke $s_{i}$ in $\mathrm{M}$ to stroke $t_{j}$ in $\mathrm{T}$ will be determined by the displacement from the two endpoints of $s_{i}$ to $t_{j}$ respectively. We first consider the displacement vector from an endpoint of $s_{i}$, say, $\overrightarrow{p_{2}}$, to $t_{j}$. Let $\vec{A}=\overrightarrow{p_{2}}-\overrightarrow{q_{1}}, \vec{B}=\overrightarrow{q_{2}}-$ $\overrightarrow{q_{1}}$, and $\theta$ be the angle between $\vec{A}$ and $\vec{B}$ (See figure 2 ). Displacement vector from point $\overrightarrow{p_{2}}$ to stroke $t_{j}$ is defined by:

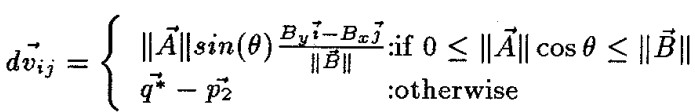

where $\overrightarrow{q^{*}}$ is the point $\overrightarrow{q_{1}}$ or $\overrightarrow{q_{2}}$ closest to $\overrightarrow{p_{2}}, \vec{i}$ and $\vec{j}$ are the basis vectors in two dimensional space.

Figure 3 gives three cases of determining displacement vector from $\overrightarrow{p_{2}}$ to stroke $t_{j}$. 


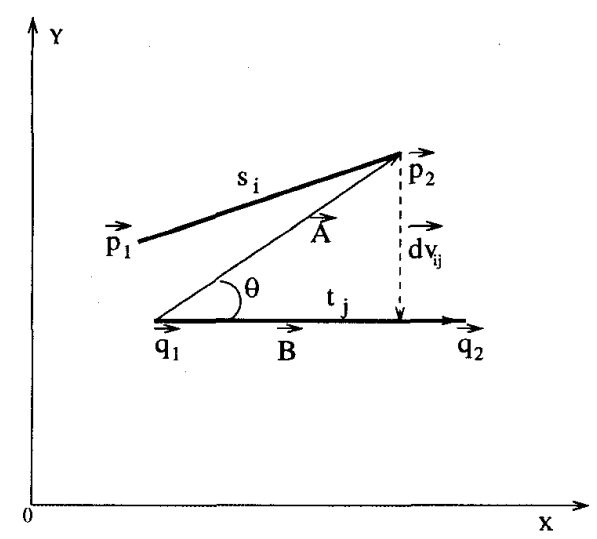

Figure 2: Geometry of displacement vector from a point to a stroke
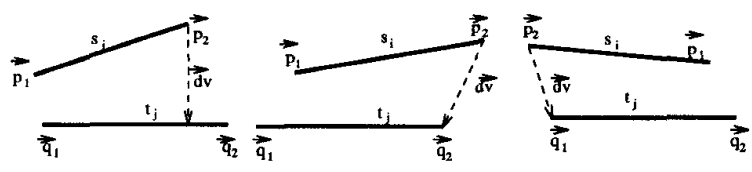

Figure 3: Three cases of DV from $\overrightarrow{p_{2}}$ to stroke $t_{j}$

DV at the endpoint $\overrightarrow{p_{1}}$ can be defined in the same way. Stroke displacement vector(SDV) from $s_{i}$ to $t_{j}$ is represented by the DVs at it's two endpoints, which will be denoted as $d \vec{v}_{i j}^{s}$ and $d \vec{v}_{i j}^{e}$ respectively. Similarity, SDV from stroke $t_{j}$ to $s_{i}$ can be defined in the same way. We will denotes them as $d \overrightarrow{v_{i j}^{r}}$ and $d \overrightarrow{v_{i j}^{r e}}$ (See figure 4 ). From physical view, these displacement vectors can be interpreted as "force" field acting on one stroke $s_{i}$, which try to distort it to be like the other stroke $t_{j}$. We can now model character $\mathrm{M}$ with elastic property, so that all of its strokes can respond some what to the "force" field, in an effort to "transform" itself to be much closer to character $T$.

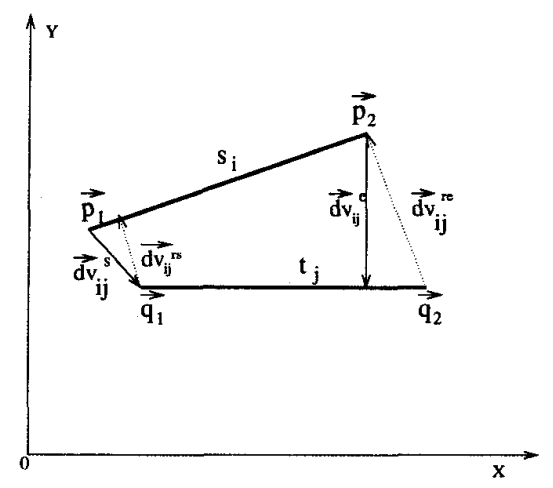

Figure 4: Stroke Displacement Vector Field

\subsection{Smoothing of Stroke Displace- ment Vectors}

As the structure of Chinese character is very complex, the whole stroke displacement vector field could be very complex too. Thus we can not determine the DV for a stroke $s_{i}$ simply by equation (1) only. In fact, all displacement vector fields at the neighbor of $s_{i}$ and $t_{j}$ should have some degree of contribution in determining the SDV for $s_{i}$. Therefore, smoothing technique is used to provide the effect that near strokes have more influence on $s_{i}$ and far strokes have less influence. First, to take into account of strokes in $\mathrm{T}$, a Gaussian weighted function is employed to smooth all stroke displacement vectors derived from $s_{i}$ to strokes in $\mathrm{T}$, at start point and end point of $s_{i}$ respectively,

$$
D \vec{V}_{i}^{s}=\gamma^{-1}\left[\frac{\sum_{j} d \overrightarrow{v_{i j}^{s}} w_{i j}^{s}}{\sum_{j} w_{i j}^{s}}-\frac{\sum_{j} d \overrightarrow{v_{i j}^{r}} w_{i j}^{r s}}{\sum_{j} w_{i j}^{r s}}\right]
$$

$$
D \vec{V}_{i}^{e}=\gamma^{-1}\left[\frac{\sum_{j} d \overrightarrow{v_{i j}^{e}} w_{i j}^{e}}{\sum_{j} w_{i j}^{e}}-\frac{\sum_{j} d \overrightarrow{v_{i j}^{r e}} w_{i j}^{r e}}{\sum_{j} w_{i j}^{r e}}\right]
$$

where

$$
w_{i j}^{s}=\exp \left\{-\frac{\left\|d \overrightarrow{v_{i j}^{s}}\right\|}{\sigma_{k}}\right\}
$$

$w_{i j}^{e}, w_{i j}^{r s}, w_{i j}^{r e}$ are given in the same way. The smoothed DV can be viewed as a function of pushing and pulling force, where the DV from $s_{i}$ to strokes in $\mathrm{T}\left(d \vec{v}_{i j}^{s}, d \vec{v}_{i j}^{e}\right)$ is interpreted as pushing force, and the DV from strokes in T to $s_{i}\left(d \overrightarrow{v_{i j}^{r}}, d \overrightarrow{v_{i j}^{r e}}\right)$ is interpreted as pulling force. Here $\gamma$ is a damping factor, with value less than 1 result in overshoot and value greater than 1 result in undershoot. $\sigma_{k}$ is the stiffness parameter at the $k$ th iteration. It has been empirically found to produce good result if the following functional form is used:

$$
\sigma_{k}=\sigma_{0} / f^{k}
$$

where $f$ is a constant in the range from 1 to 2 .

Furthermore, to take into account the displacement vector field derived from the neighbors of stroke $s_{i}, D \vec{V}_{i}^{s}$ and $D \vec{V}_{i}^{e}$ will be smoothed again by the following equation so as to produce the final stroke displacement vector (SDV) for $s_{i}$,

$$
S \overrightarrow{D V}_{i}^{s}=\frac{\sum_{j} D \vec{V}_{j}^{s} G_{j}^{s} N_{i j}}{\sum_{j} G_{j}^{s}}
$$




$$
S \overrightarrow{D V_{i}^{e}}=\frac{\sum_{j} D \vec{V}_{j}^{e} G_{j}^{e} N_{i j}}{\sum_{j} G_{j}^{e}}
$$

where:

$$
\begin{aligned}
G_{j}^{s} & =\exp \left\{\left\|D \vec{V}_{j}^{s}\right\| / \tau\right\} \\
G_{j}^{e} & =\exp \left\{\left\|D \vec{V}_{j}^{e}\right\| / \tau\right\} \\
N_{i j} & = \begin{cases}1 & \text { if } s_{j} \text { is a neighbor of } s_{i} \\
0 & \text { otherwise }\end{cases}
\end{aligned}
$$

We found that equation (4) and (5) are essential for keeping the topological structure of a character during deformation.

\subsection{Iterative Elastic Matching Process}

Elastic matching a model character $M$ with an unknown character $\mathrm{T}$ is performed by deforming the model $M$ according to its SDVs obtained, step by step, to "transform" M much closer to T. Deformation of $\mathrm{M}$ is performed through the following equation:

$$
\left\{s_{i}^{t+1}\right\}=\left\{s_{i}^{t}\right\}+\left\{S \overrightarrow{D V}_{i}^{s^{t}}, S \overrightarrow{D V}_{i}^{e^{t}}\right\}
$$

$t$ stands for the iteration number. Iteration stop criteria is:

$$
\frac{1}{m} \sum_{i}^{m}\left[\left\|S \overrightarrow{D V_{i}^{s}}{ }^{t}\right\|+\left\|S \overrightarrow{D V_{i}^{e}} e^{t}\right\|\right] \leq \varepsilon
$$

where $\varepsilon$ is a predefined small value.

Matching similarity between $\mathrm{M}$ and $\mathrm{T}$ is measured by:

$$
\begin{aligned}
\text { Dissimilarity }= & \frac{1}{m} \sum_{i}^{m}\left[\left\|\overrightarrow{d v_{i}^{s}}\right\|+\left\|d \vec{v}_{i}^{e^{*}}\right\|\right] \\
& +\frac{1}{n} \sum_{j}^{n}\left[\left\|d \vec{v}_{j}^{s^{*}}\right\|+\| \overrightarrow{\left.d \vec{v}_{j}^{e^{*}} \|\right]}\right.
\end{aligned}
$$

where:

$$
\left\|d \vec{v}_{i}^{s}\right\|=\min _{j}\left\{\left\|d \overrightarrow{v_{i j}^{s}}\right\|\right\}
$$

$\left\|d \vec{v}_{i}^{e^{*}}\right\|,\left\|d \vec{v}_{j}^{s^{*}}\right\|,\left\|d \vec{v}_{j}^{e^{*}}\right\|$ are defined in the same way.

\section{Experiments}

200 categories of frequently used Chinese characters are used in our experiments. 30 persons are asked to write each of the 200 character. Each handwritten character is scanned and normalized to $64 \times 64$ image pattern. A stroke extractor is then employed to extract all strokes from the skeleton of the character[12].

Two characters from each category are selected as the flexible templates, others are used as testing characters. When an unknown character is input, each template is matched with it by the elastic matching algorithm proposed. Figure 5 shows the iterative elastic matching process of two Chinese character "No", figure $5(\mathrm{a})$ is the input character, $5(\mathrm{~b})$ is the flexible template, $5(\mathrm{c})$ shows that after several iterations of deformation; the template has become very similar to the input character. Figure 6 gives another example.

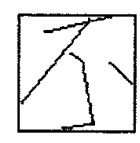

[a]

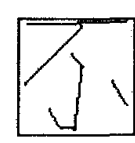

(b)
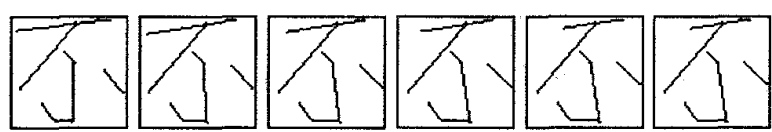

[c]

Figure 5: Elastic matching process of two Chinese characters 'no'. (a). Input character. (b). The flexible model character. (c). The elastic matching process. Note that after seven iterations, the model character has been deformed to be very like the input character.
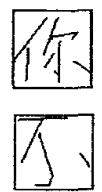

[a]
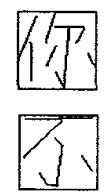

(b)
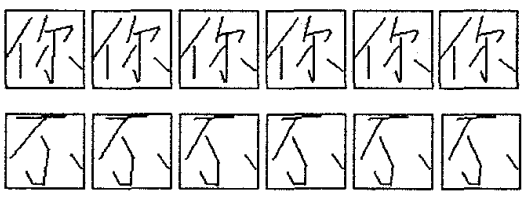

(c)
Figure 6: (a). Input characters. (b). The flexible models. (c). Deformation and elastic matching process.

After all templates have been matched with the unknown character, the one which gives the best match (with the minimal dissimilarity value) is picked up as the recognition result. Table 1 gives the obtained recognition rate for 100 and 200 categories of handwritten Chinese characters respectively, here each category contains 28 testing characters. 


\begin{tabular}{l|c}
\hline Datum & Recognition Rate \\
\hline $100 \times 28$ & $96.6 \%$ \\
\hline $200 \times 28$ & $94.9 \%$ \\
\hline
\end{tabular}

Table 1: Experimental results of recognizing 100 and 200 categories of HCCs, each category contains 28 variations of testing characters

\section{Conclusion}

In this paper, we have proposed a deformable elastic matching model for handwritten Chinese character recognition. HCC is regarded as a kind of deformable objects. Distortion among different handwriting characters is modelled by a set of Stroke Displacement Vectors. Encouraging results were obtained.

Extension of this method can be applied to line drawings matching and shape recognition as well.

One disadvantage of the elastic matching model is that if the global misalignment between two image patterns is too large, it's difficult to determine the correct Displacement Vectors and thus the matching scheme may fail. In such situation, deforming an object under some constraints(such as some topologicalinvariant features of the object) may merit further study.

\section{Acknowledgement}

We would like to acknowledge support from the Hong Kong research grant council.

\section{References}

[1] B. Widrow, "The Rubber Mask Technique," Part one and Part two, Pattern Recognition, vol. 5, pp. 175-211, 1973.

[2] D.J. Burr, "Elastic Matching of Line Drawings",IEEE Trans on Pattern Anal,, Machine Intell., vol-3, No. 6, pp. 709-713, 1981.

[3] D. Terzopoulos, A. Witkin, and M. Kass, "Energy constraints on Deformable Models: Recovering Shape and Non-rigid Motion", in Proceedings, $A A A I$ 87, vol. 2, pp. 755-760, July, 1987.
[4] M. Kass, A. Witkin and D. Terzopouls, "SNAKE: Active Contour Model", in Proceedings Int. Conference Comput. Vision. pp. 259$268,1987$.

[5] A.Hill, T.F. Cootes and C;J. Taylor, "A Generic System for Image Interpretation Using Flexible Templates", in Proceedings of British Machine Vision Conf, pp. 276-285, 1992.

[6] T.F. Cootes, C.J. Taylor, D.H. Cooper and J.Graham, "Training Models of Shape From Sets of Examples", in Proceedings of British Machine Vision Conf, pp. 9-18, 1992.

[7] Ruzena Rajcsy, "Multiresolution Elastic Matching", Computer Vision, Graphics, and Image Processing, Vol 46, No 1, pp. 1-21, 1989.

[8] Mehran Moshfeghi, Surendra Ranganath, and Ken Nawyn, "Three-Dimensional Elastic Matching of Volumes", IEEE Trans. on Image Processing, vol. 3, no. 2, pp128-138, 1994.

[9] Shunji Mori, C. Y. Suen and Kazuhiko Yamamoto, "Historical Review of OCR Research and Development", Proceedings of the IEEE, Vol. 80, No.7, July, 1992.

[10] Jean-Michel Bertille, "An Elastic Matching Approach Applied to Digit Recognition", in Proceedings of the Second International Conf. on Document Anal. and Processing, pp. 82-85, 1993.

[11] Toru Wakahara, "Shape Matching using LAT and its Application to Handwritten Numeral Recognition", IEEE Trans. on PAMI, Vol. 16, No. 6, pp. 618 - 629, 1994.

[12] Fang-Hsuan Cheng and Wen-Hsing Hsu, "Three Stroke Extraction Methods for Recognition of Handwritten Chinese Characters", in Proceedings of the 1986 International Conference on Chinese Computing, pp. 191-195, 1986. 